\title{
Contraintes résiduelles et comportement mécanique de revêtements nickel-bore
} \author{
Bemporad $^{3}$, Alain Iost ${ }^{1}$, et Mariana Henriette Staia ${ }^{1}$ \\ ${ }^{1}$ MSMP EA-7350, Arts et Métiers ParisTech, 8 boulevard Louis XIV, Lille, France \\ ${ }^{2}$ Faculté d'Ingénierie, Université de Mons, 56 rue de l'Epargne, Mons, Belgique \\ ${ }^{3}$ Engineering Department, Roma Tre University, 79 via della Vasca Navale, Rome, Italie
}

Alex Montagne ${ }^{1, *}$, Véronique Vitry ${ }^{2}$, Luiza Bonin ${ }^{2}$, Muhammad Zeeshan Mughal $^{3}$, Marco Sebastiani ${ }^{3}$, Edoardo

Reçu le 30 novembre 2018 / Accepté le 14 mars 2019

\begin{abstract}
Résumé. Deux revêtements à base nickel obtenus par des bains réducteurs, l'un brut et l'autre ayant subi un traitement thermique sont étudiés. L'essai d'indentation a été mis en œuvre pour caractériser la dureté et le module de Young de ces dépôts. Les résultats montrent une bonne homogénéité des propriétés mécaniques dans l'épaisseur du revêtement, alors que le traitement thermique a eu pour effet d'augmenter sa dureté et son module de façon significative. Les contraintes résiduelles dans les revêtements sont étudiées par la méthode du micro anneau incrémental. Les contraintes calculées sont faibles, dispersées et ne semblent pas dépendre du traitement thermique. En l'absence de contraintes résiduelles avant et après traitement thermique, les variations de propriétés mécaniques ont été attribuées à la modification microstructurale du revêtement et à la précipitation de phases dures.
\end{abstract}

Mots clés : revêtements nickel / dureté / module de Young / nanoindentation / contraintes résiduelles

\begin{abstract}
Residual stresses and mechanical behavior of nickel-boron coatings. Two nickel-based coatings obtained from electroless plating baths are studied. The first one is studied as-deposited whereas the second one was subjected to a heat-treatment. Hardness and Young's modulus of coatings have been investigated by means of indentation tests. Results show that mechanical properties are homogenous in the whole thickness of the coating. On the other hand, the heat treatment leads on a significantly increase of hardness and modulus. Residual stresses in the coatings are studied by the incremental ring core method. Values of residual stresses are small, scattered and do not seem to be heat-treatment dependent. Without any modifications of residual stresses before and after heat treatment, mechanical properties evolutions were attributed to the microstructural modification of the coating and to the precipitation of hard phases.
\end{abstract}

Keywords: nickel coatings / hardness / Young's / modulus / nanoindentation / residual stresses

\section{Introduction}

Les revêtements à base nickel connaissent actuellement un regain d'intérêt. Ils sont pressentis pour remplacer les revêtements à base de chrome hexavalent qui présentent des risques environnementaux élevés et sont désormais interdits par la réglementation européenne [1]. Les applications de ces revêtements sont multiples du fait de leur dureté élevée et de leur bonne résistance à la corrosion et à l'usure. Leur facilité de dépôt sur des pièces de formes complexes a également contribué à leur popularité [2-5]. Les propriétés mécaniques (dureté, résistance à l'usure) sont intimement liées aux contraintes résiduelles présentent dans le revêtement ou à l'interface avec le substrat

\footnotetext{
* e-mail: alex.montagne@ensam.eu
}

$[6,7]$. En particulier, des contraintes de tension favorisent la propagation de fissures et donc une diminution de la ténacité ainsi qu'une diminution de la dureté; des contraintes de compression ont l'effet inverse.

La détermination des contraintes résiduelles dans les revêtements n'est pas toujours évidente. La diffraction des rayons $\mathrm{X}$ offre une résolution spatiale submicronique à condition que la structure soit cristalline [8]; la méthode de Stoney, qui lie la courbure d'un échantillon aux contraintes résiduelles, nécessite une forme particulière du substrat ou un amincissement contrôlé du revêtement [9]. Les essais de nanoindentation peuvent, eux-aussi, permettre d'estimer les contraintes résiduelles en comparant les courbes forcedéplacement obtenues sur matériaux contraints et totalement relaxés, mais ce dernier état peut être difficile à obtenir en termes de microstructure [10]. 
Dans cette étude, nous proposons de mettre en application la méthode de l'anneau incrémental (Ring core method) inspirée de la méthode du trou incrémental classiquement utilisée en mécanique [6,11-13]. Le principe consiste à relaxer complètement une partie du matériau par un retrait de matière progressif et à suivre l'évolution de la déformation de surface. Le retrait de la matière se fait par un faisceau d'ions focalisés (Focused ions beam [FIB]) qui creuse un anneau autour de la zone de référence qui est observée à chaque incrément de profondeur. Les images prises à chaque étape sont analysées par corrélation d'images [11]. Une grille de points de référence est apposée à la surface et le mouvement relatif de chacun de ces points est suivi d'une étape à l'autre, ce qui permet ainsi de déterminer la déformation de la surface. D'après les travaux de Korsunsky et al. [12], l'évolution de la déformation en fonction de la profondeur de forage est une courbe asymptotique et la matière est totalement relaxée quand le rapport diamètre/profondeur de forage est égal à 1. Ces auteurs proposent, pour un état de contrainte équi-biaxial, une modification de la relation de Hooke:

$$
\sigma=\frac{-E \Delta \varepsilon}{1-v}
$$

où $\sigma$ est la contrainte résiduelle, $E$ le module de Young du matériau, $v$ son coefficient de Poisson et $\Delta \varepsilon$ l'incrément de déformation entre l'état initial (contraint) et l'état final (complètement relaxé).

\section{Matériaux et protocole expérimental}

Des tôles fines d'acier S235JR (1 mm) ont été sélectionnées comme substrat. Avant la réalisation du dépôt de nickelbore, ces tôles ont été mises à dimensions $25 \times 25 \mathrm{~mm}^{2}$, polies jusqu'au papier abrasif SiC grade 1200 (diamètre des grains de $14 \mu \mathrm{m}$ ), dégraissées à l'acétone, puis activées dans une solution d'acide chlorhydrique à $30 \%$ en volume avant immersion dans le bain utilisé pour le dépôt.

Le nickel-bore (NiB) est déposé suivant la méthode développée par Delaunois et al. [11]. La solution est composée de chlorure de nickel hexahydraté, complexé à l'éthylène diamine (source d'ions métalliques), de borohydrure de sodium (agent réducteur), de tungstate de plomb en très faible quantité (agent stabilisant). Le pH est maintenu dans la gamme de travail (supérieur à 12) par une concentration importante en hydroxyde de sodium.

Les conditions de travail pour produire les échantillons sont une température de $95{ }^{\circ} \mathrm{C}\left( \pm 1^{\circ} \mathrm{C}\right)$, un rapport surface/ volume de solution de $25 \mathrm{~cm}^{2} . \mathrm{L}^{-1}$ et une agitation mécanique (via une puce magnétique) constante. Avec de telles conditions, l'épaisseur des revêtements est de $20 \mu \mathrm{m}$.

Après dépôt, les échantillons sont rincés à l'eau déminéralisée, puis séchés à l'air chaud. Une partie d'entre eux subit un traitement thermique d'une heure sous atmosphère légèrement réductrice $\left(95 \% \mathrm{Ar}-5 \% \mathrm{H}_{2}\right)$ à une température de $400^{\circ} \mathrm{C}$. Ces conditions de travail qui apportent les meilleures propriétés mécaniques aux revêtements ont été sélectionnées sur la base de résultats obtenus lors de précédents travaux [12-14].
Les essais d'indentation sont effectués à l'aide d'un nanoindenteur G200 (MTS) équipé d'un indenteur Berkovich en mode Continuous stiffness measurement (CSM) avec une pénétration maximale imposée de $300 \mathrm{~nm}$. La pointe a été étalonnée par une série d'indentation dans un matériau de référence dont le module de Young est connu (silice fondue, $\mathrm{E}=72 \mathrm{GPa}$ ) et les courbes d'indentation force-déplacement ont été analysées en suivant la méthode d'Oliver et Pharr [15].

La méthode de l'anneau incrémental consiste à creuser un anneau autour d'une zone de référence, ici un pilier de $25 \mu \mathrm{m}$ de diamètre, dont la déformation est observée par imagerie en microscopie électronique en balayage (MEB) $[16,17]$. Le forage est effectué en 60 étapes qui correspondent chacune à un enlèvement de matière sur une profondeur d'environ $0,4 \mu \mathrm{m}$. Afin de limiter les artefacts de mesure, dix images sont prises pour chaque étape, puis analysées par corrélation d'images [16].

Les échantillons observés en microscopie électronique à transmission (MET) sont préparés par découpe FIB, dans un équipement FEI Quanta 2003D dual beam, perpendiculairement à la surface libre, puis analysés au moyen d'un MET Philips CM 20 sous une tension de $200 \mathrm{kV}$.

\section{Résultats}

Pour étudier leurs propriétés mécaniques, les revêtements sont enrobés dans une résine à froid (DuroFast, Struers), polis mécaniquement par différents papiers $\mathrm{SiC}$ (du grade 80 au 4000), puis finalement avec une solution collö̈dale d'alumine (OPS, Struers). Les réseaux d'indentation effectués sur les coupes transverses des échantillons sont positionnés de sorte à couvrir le substrat (acier), le revêtement (nickel) et l'enrobage (résine).

Suivant les recommandations de Yetna et al. [18], pour qui un rapport profondeur résiduelle $\left(h_{\mathrm{p}}\right)$ sur profondeur maximale $\left(h_{\max }\right)$ supérieur à 0,83 est en faveur de la formation d'un bourrelet autour de l'empreinte, les courbes force-déplacement de l'acier $\left(h_{\mathrm{p}} / h_{\max }=0,9\right)$ devraient être dépouillées par le modèle de Loubet et al. [19]. Par contre, selon le même critère, le revêtement nickel-bore subit bien un enfoncement élastique autour du contact pointe-surface $\left(h_{\mathrm{p}} / h_{\mathrm{max}}=0,7\right)$ et doit être analysé avec le modèle d'Oliver et Pharr [15]. Comme ici, l'objet d'étude n'est pas l'acier mais le revêtement, toutes les courbes sont analysées par le même modèle d'Oliver et Pharr, et par conséquence, la dureté et le module du substrat sont surestimés à cause de la présence des bourrelets.

La figure 1 présente l'évolution de dureté et de module de Young dans les revêtements $\mathrm{NiB}$ brut et traité thermiquement, en fonction de la distance à l'interface avec le substrat, prise comme origine. Les traits continus rouges représentent la moyenne des valeurs et les lignes en pointillé l'écart-type. Tout d'abord, pour un même échantillon, aucune évolution de propriétés mécaniques n'est observée dans l'épaisseur du revêtement que ce soit pour la dureté ou le module de Young, traduisant l'homogénéité du dépôt et ce sans ou avec traitement thermique. En revanche, le traitement thermique a eu pour effet d'augmenter la dureté et le module de Young du 

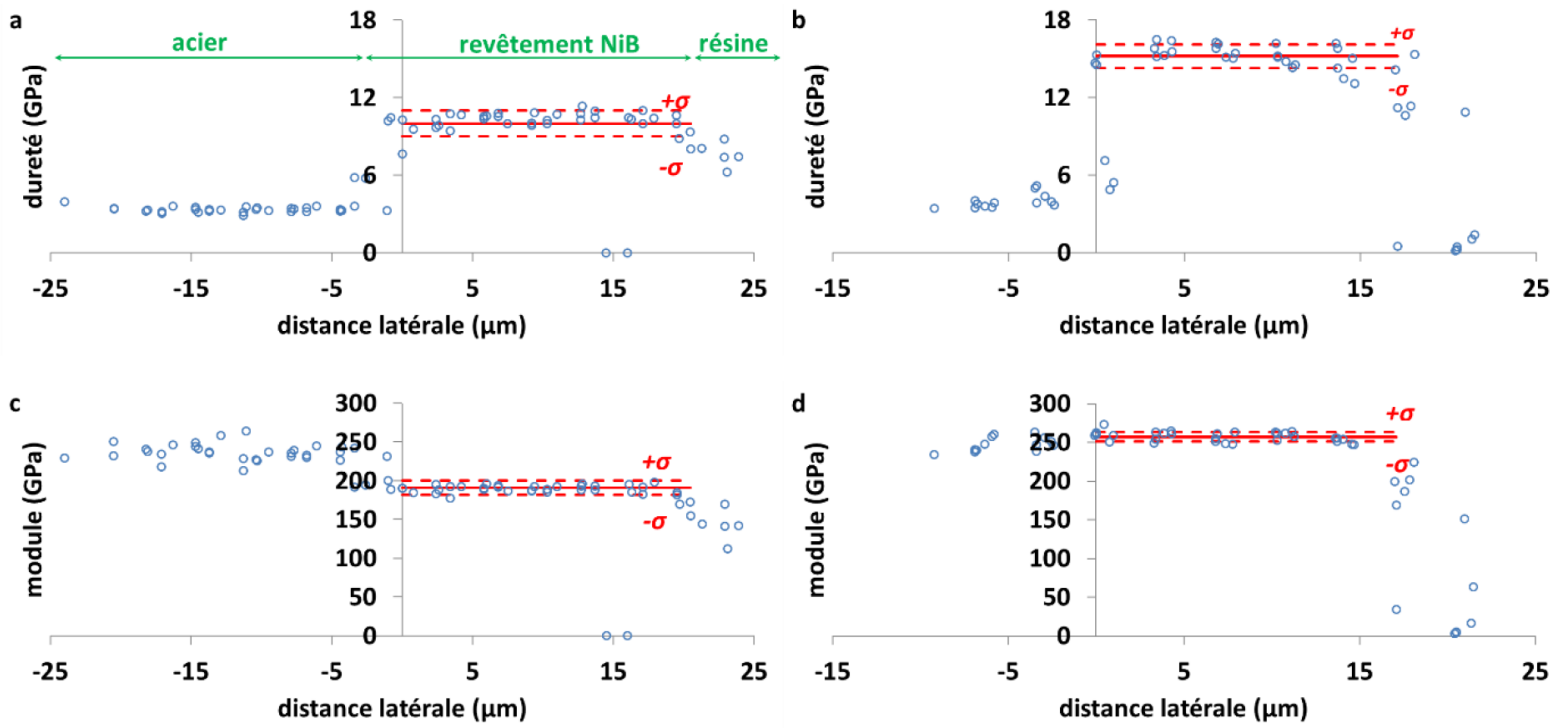

Fig. 1. Dureté (a) et module (c) de l'échantillon brut, dureté (b) et module (d) de l'échantillon traité thermiquement. La moyenne des valeurs est représentée (trait rouge continu), ainsi que l'écart-type (trait rouge pointillé).

Fig. 1. Hardness (a) and modulus (c) of the pristine sample, hardness (b) and modulus (d) of the heat treated sample. The average value is drawn (continuous red line), as well as the standard deviation (dashed red line).

Tableau 1. Valeurs de dureté et de module pour les revêtements nickel-bore sans (brut) et avec traitement thermique (TT), les valeurs sont assorties de leur écarttype.

Table 1. Hardness and modulus for nickel-bore coatings without (pristine) and with heat treatment (TT), standard deviations are also reported.

\begin{tabular}{lll}
\hline & NiB brut & NiB TT \\
\hline$H(\mathrm{GPa})$ & $10 \pm 1$ & $15,2 \pm 0,9$ \\
$E(\mathrm{GPa})$ & $191 \pm 9$ & $256 \pm 6$ \\
\hline
\end{tabular}

revêtement. Le tableau 1 reprend ces valeurs pour le revêtement de nickel. Le traitement thermique n'a pas d'effet sur le comportement sous indentation de l'acier.

La figure 2 représente l'évolution de la déformation de surface en fonction de la profondeur de forage pour les deux revêtements $\mathrm{NiB}$. Les valeurs de relaxation sont basses, ce qui implique que les contraintes résiduelles sont extrêmement faibles avec ou sans traitement thermique. Pour l'échantillon brut (Fig. 2a), les valeurs finales de la relaxation indiquent des contraintes pouvant être de compression (essai T1, Fig. 2a) ou de tension (essai T3, Fig. 2a) selon la zone étudiée. La répartition des contraintes est donc hétérogène dans le matériau. En regardant plus en détail le test T2 (Fig. 2a), l'évolution non monotone de la relaxation confirme l'hétérogénéité des contraintes, avec en début de relaxation l'effet d'une contrainte de traction pour tendre vers une contrainte globale nulle. La distribution des contraintes est hétérogène dans le plan du revêtement mais également dans son épaisseur.
Les courbes de relaxation s'écartent du modèle proposé par Korsunsky et al. [20]. En particulier pour les tests T1 et T2 du revêtement brut, la relaxation ne présente pas de valeur asymptotique très prononcée (Fig. 2a). En première analyse, les contraintes résiduelles des revêtements sont calculées à partir des valeurs de relaxation à la profondeur de forage maximale et sont répertoriées dans le tableau 2. Les contraintes résiduelles restent faibles (moins de $200 \mathrm{MPa}$ en valeur absolue) et ne sont pas affectées par le traitement thermique.

Nous proposons d'examiner plus finement l'hétérogénéité des contraintes résiduelles calculées. Pour cela, la relaxation de la surface n'est pas étudiée sur l'ensemble du pilier de $25 \mu \mathrm{m}$ mais sur des zones plus restreintes qui correspondent à des «bouquets» de la structure chou-fleur. Les zones isolées sont représentées sur la figure 3, extraites de l'essai T3 du revêtement brut. L'évolution de la déformation dans ces zones est présentée sur la figure 4. De nouveau, la dispersion importante montre que les contraintes résiduelles sont faibles et dispersées au sein même d'un pilier de $25 \mu \mathrm{m}$ de diamètre.

Les contraintes résiduelles dans les revêtements semblent présentes à de très faibles échelles et avec une très forte hétérogénéité. Les zones sous tension sont accommodées par les zones en compression, de sorte qu'à plus grande échelle la contrainte résiduelle est globalement nulle. De plus, la présence des interfaces entre les «bouquets » de la structure doit vraisemblablement contribuer à la relaxation des contraintes.

Les variations de contraintes résiduelles entre l'échantillon vierge et celui traité thermiquement sont faibles (Tab. 1) et ne peuvent expliquer les évolutions importantes de dureté observées (Fig. 1). Dès lors, les variations de 

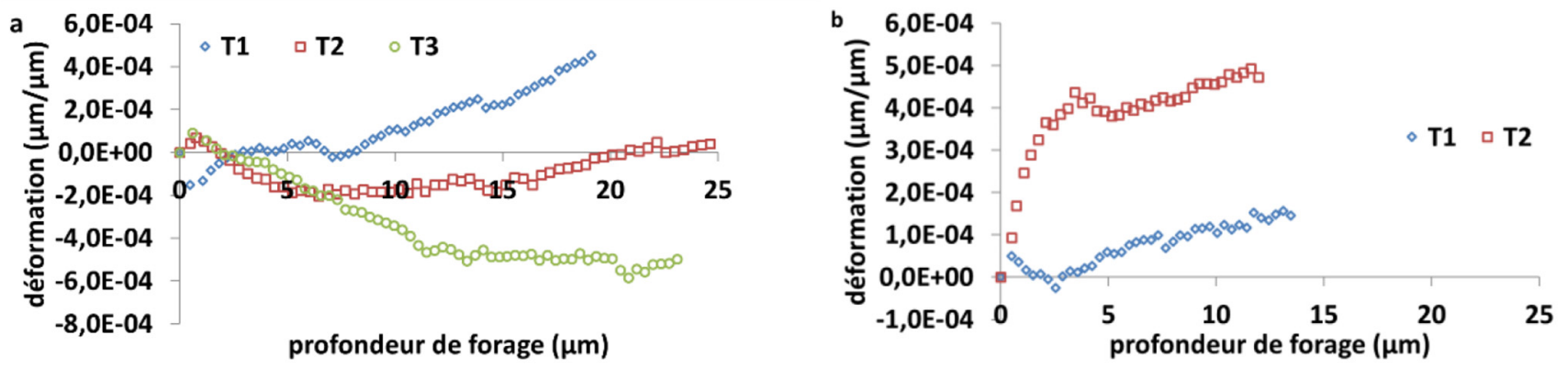

Fig. 2. Évolution de la relaxation de surface en fonction de la profondeur de forage pour les revêtements nickel-bore (a) brut et (b) traité thermiquement. Les « $\mathrm{T}$ » se rapportent à différents piliers du même échantillon.

Fig. 2. Evolution of surface relaxation as a function of drilling depth for nickel-boron (a) pristine and (b) heat-treated coatings. The "T" refer to different pillars from the same sample.

Tableau 2. Valeurs de relaxation $\varepsilon$ pour la profondeur de forage maximale et contrainte résiduelle correspondante $\sigma$ pour les revêtements nickel-bore sans (brut) et avec (TT) traitement thermique.

Table 2. Relaxation values $\varepsilon$ at maximum drilling depth and corresponding residual stress $\sigma$ for nickel-bore coatings without (pristine) and with (TT) heat treatment.

\begin{tabular}{|c|c|c|c|c|c|}
\hline \multicolumn{3}{|c|}{ NiB brut } & \multicolumn{3}{|c|}{$\underline{\mathrm{NiB} T \mathrm{~T}}$} \\
\hline Test & $\varepsilon\left(\times 10^{4}\right)$ & $\sigma(\mathrm{MPa})$ & Test & $\varepsilon\left(\times 10^{4}\right)$ & $\sigma(\mathrm{MPa})$ \\
\hline $\mathrm{T} 1$ & 4,5 & -122 & $\mathrm{~T} 1$ & 4,8 & -176 \\
\hline $\mathrm{T} 2$ & 0,4 & -11 & $\mathrm{~T} 2$ & 1,5 & -55 \\
\hline T3 & $-5,2$ & 141 & - & - & - \\
\hline
\end{tabular}

dureté et de module doivent être rapprochées de la microstructure des revêtements.

La structure et la microstructure des dépôts de nickelbore synthétisés par la méthode sélectionnée, ainsi que les méthodes utilisées pour leur étude, ont été largement décrites dans divers articles $[14,21,22]$. Ces dépôts contiennent $5 \%$ en masse de bore, environ $1 \%$ de plomb et près de $94 \%$ de nickel. La présence de plomb est liée à l'utilisation des sels de ce métal nécessaires à la stabilisation du bain. Malgré les faibles teneurs présentes en solution, le plomb plus noble que le nickel sera réduit conjointement à celui-ci lors de la synthèse.

Les revêtements préparés par réduction chimique, et en particulier les dépôts de nickel-bore, présentent une croissance colonnaire qui est à l'origine de la morphologie de surface particulière en «chou-fleur » clairement observable sur la figure 3. La présence et la taille des colonnes est directement liée à la nature et à la quantité d'agent stabilisant utilisé [21,23].

Les dépôts obtenus par réduction chimique possèdent une structure hors-équilibre. En effet, pour les teneurs en bore concernées, la cristallisation du nickel métallique est fortement inhibée par la présence d'atomes de bore ainsi que par la précipitation de borures de nickel prévus par le diagramme de phases $\mathrm{Ni}-\mathrm{B}$. Les revêtements sont des solutions solides très sursaturées de bore dans le nickel. Elles présentent un ordre à courte distance mais ne peuvent être considérées comme cristallines [24] ainsi qu'on peut l'observer sur les figures 5a et 5c (coupe MET d'un échantillon après dépôt et cliché de diffraction électronique) du même échantillon.

Le traitement thermique à $400^{\circ} \mathrm{C}$ apporte une énergie suffisante à la cristallisation des dépôts. On observe, après traitement, la formation de borure de nickel $\left(\mathrm{Ni}_{3} \mathrm{~B}\right)$ nanocristallin, avec une taille de grains de l'ordre de $50 \mathrm{~nm}$ qui correspond à la structure prévue par le diagramme de phase. Ces cristaux sont clairement visibles sur la figure 5b. Cette phase présente une structure orthorhombique Pnma avec 12 atomes de nickel et 4 de bore par maille élémentaire, similaire à la structure de la cémentite $\mathrm{Fe}_{3} \mathrm{C}$ comme l'indique le cliché de diffraction électronique de la figure $5 \mathrm{~d}$. Il s'agit donc d'une phase très dure, dont les propriétés sont encore renforcées par sa nature nanocristalline dans les revêtements.

\section{Conclusions}

Dans cette étude, les revêtements nickel-bore obtenus par des solutions réductrices présentent une structure classique en « chou-fleur ». En dépit de la microstructure hétérogène, les propriétés mécaniques obtenues par indentation (dureté et module de Young) sont constantes dans l'épaisseur du revêtement. Le traitement thermique appliqué au revêtement a pour conséquence une augmentation de la dureté et du module de Young de respectivement 50 et $35 \%$. La 

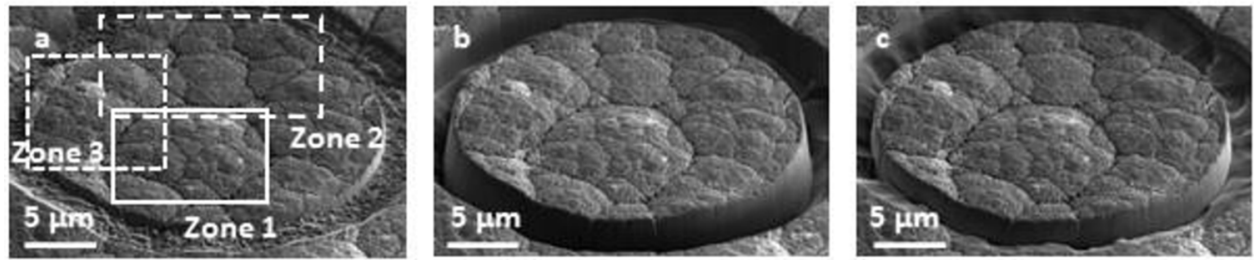

Fig. 3. Pilier avec un forage à a) $1,2 \mu \mathrm{m}$, b) $3,6 \mu \mathrm{m}$ et c) $8,5 \mu \mathrm{m}$ pour le revêtement nickel-bore sans traitement thermique.

Fig. 3. Drilling depth step at a) $1.2 \mu \mathrm{m}$, b) $3.6 \mu \mathrm{m}$ and c) $8.5 \mu \mathrm{m}$ for nickel-boron coating without heat treatment.

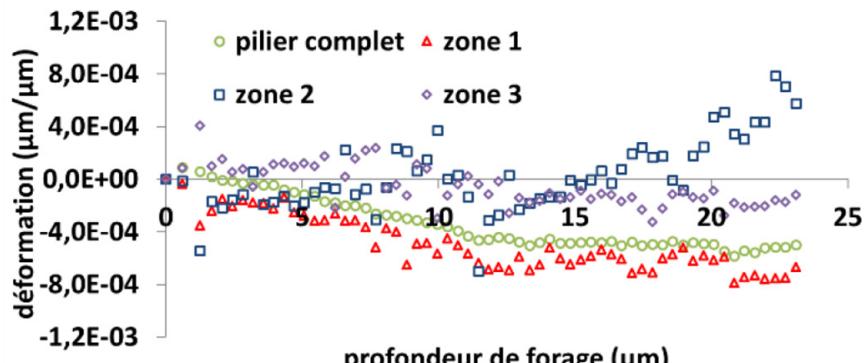

Fig. 4. Évolution des relaxations en fonction de la profondeur de forage pour l'ensemble du pilier et pour les zones 1 à 3 indiquées sur la figure 3a.

Fig. 4. Relaxations evolution as a function of drilling depth for the whole pillar and areas 1 to 3 reported on Figure $3 a$.

méthode de l'anneau incrémental a permis de montrer que les contraintes résiduelles, avant et après traitement thermique, sont faibles (inférieures à $200 \mathrm{MPa}$ en valeur absolue), dispersées et locales. La structure typique en chou-fleur, liée à la croissance colonnaire de ce type de revêtement, semble être à l'origine de la relaxation des contraintes entre les différentes zones du matériau. L'évolution des propriétés mécaniques est alors attribuée à la formation de phases dures lors du traitement thermique.

Remerciements. A. Montagne souhaite remercier la Fondation Arts et Métiers pour avoir soutenu financièrement son séjour au sein du département d'ingénierie de l'université de Roma Tre.

\section{References}

1. Directive 2011 /65/UE du Parlement européen et du Conseil du 8 juin 2011 relative à la limitation de l'utilisation de certaines substances dangereuses dans les équipements électriques et électroniques

2. A. Brenner, G.E. Riddell, Nickel plating on steel by chemical reduction, J. Res. NBS 37(1), 31 (1946)

3. P. Sahoo, S.K. Das, Tribology of electroless nickel coatings A review, Mater. Des. 32(4), 1760 (2011)

4. V. Vitry, A.-F. Kanta, F. Delaunois, Mechanical and wear characterization of electroless nickel-boron coatings, Surf. Coat. Technol. 206(7), 1879 (2011)
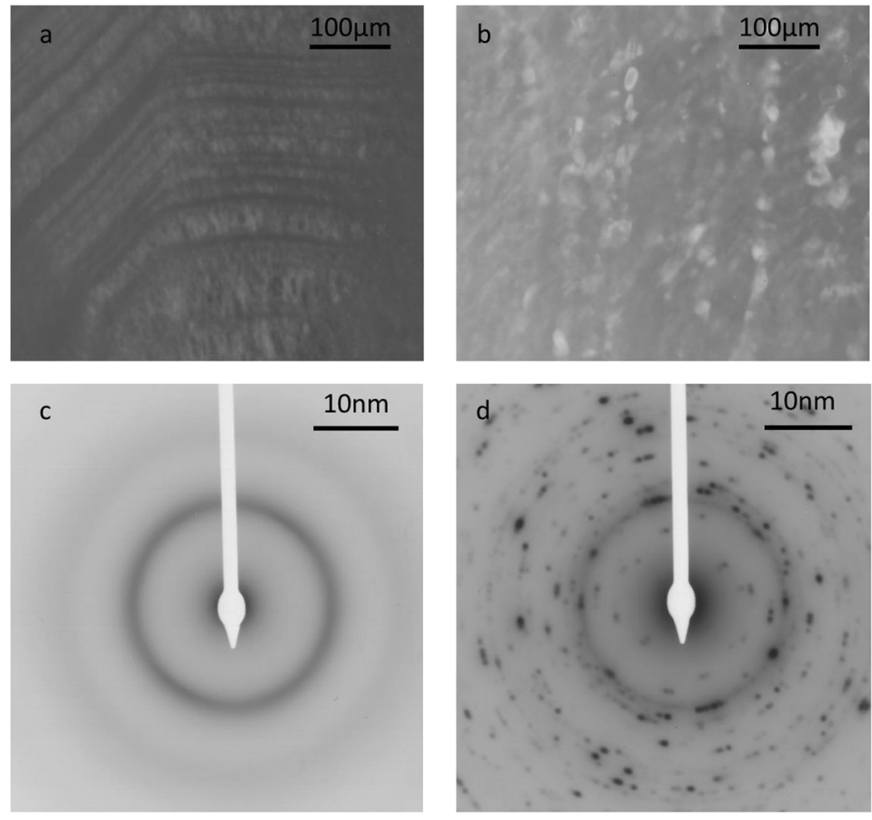

Fig. 5. Micrographies en microscopie électronique à transmission d'un revêtement de nickel-bore a) brut, b) après traitement thermique. Cliché de diffraction électronique obtenu pour un échantillon de nickel-bore c) brut, d) après traitement thermique.

Fig. 5. Transmission electron micrographs of a nickel-boron coating a) pristine, b) after heat treatment. Electron diffraction pattern obtained for a nickel-boron sample c) pristine, d) after heat treatment.

5. J. Sudagar, J. Lian, W. Sha, Electroless nickel, alloy, composite and nano coatings - A critical review, J. Alloys Compd. 571, 183 (2013)

6. M. Sebastiani, E. Bemporad, F. Carassiti, On the influence of residual stress on nano-mechanical characterization of thin coatings, J. Nanosci. Nanotechnol. 11(10), 8864 (2011)

7. H.K. Tönshoff, B. Karpuschewski, A. Mohlfeld, H. Seegers, Influence of stress distribution on adhesion strength of sputtered hard coatings, Thin Solid Films 332(1-2), 146 (1998)

8. A.M. El-Sherik, J. Shirokoff, U. Erb, Stress measurements in nanocrystalline Ni electrodeposits, J. Alloys Compd. 389(12), $140(2005)$

9. P.J. Withers, H.K.D.H. Bhadeshia, Residual stress. Part 1Measurement techniques, Mater. Sci. Technol. 17(4), 355 (2001) 
10. J. Jang, Estimation of residual stress by instrumented indentation: a review, J. Ceram. Process. Res. 10(3), 391 (2009)

11. F. Delaunois, J.P. Petitjean, P. Lienard, M. Jacob-Duliere, Autocatalytic electroless nickel-boron plating on light alloys, Surf. Coat. Technol. 124(2-3), 201 (2000)

12. F. Delaunois, P. Lienard, Heat treatments for electroless nickel-boron plating on aluminium alloys, Surf. Coat. Technol. 160(2-3), 239 (2002)

13. A.-F. Kanta, V. Vitry, F. Delaunois, Effect of thermochemical and heat treatments on electroless nickel-boron, Mater. Lett. 63(30), 2662 (2009)

14. V. Vitry, F. Delaunois, C. Dumortier, Mechanical properties and scratch test resistance of nickel-boron coated aluminium alloy after heat treatments, Surf. Coat. Technol. 202(14), 3316 (2008)

15. W.C. Oliver, G.M. Pharr, An improved technique for determining hardness and elastic modulus using load and displacement sensing indentation experiments, J. Mater. Res. 7, 1564 (1992)

16. M. Sebastiani, C. Eberl, E. Bemporad, G.M. Pharr, Depth-resolved residual stress analysis of thin coatings by a new FIB-DIC method, Mater. Sci. Eng. A 528(27), 7901 (2011)

17. A.M. Korsunsky, M. Sebastiani, E. Bemporad, Focused ion beam ring drilling for residual stress evaluation, Mater. Lett. 63(22), 1961 (2009)
18. M. Yetna N'Jock, et al., Un critère simple d'identification du mode de déformation par indentation, Mater. Tech. 103(6), $603(2015)$

19. J.L. Loubet, J.M. Georges, O. Marchesini, G. Meille, Vickers indentation curves of magnesium oxide $(\mathrm{MgO})$, J. Tribol. 106(1), 43 (1984)

20. A.M. Korsunsky, M. Sebastiani, E. Bemporad, Residual stress evaluation at the micrometer scale: Analysis of thin coatings by FIB milling and digital image correlation, Surf. Coat. Technol. 205(7), 2393 (2010)

21. V. Vitry, A. Sens, A.-F. Kanta, F. Delaunois, Experimental study on the formation and growth of electroless nickel-boron coatings from borohydride-reduced bath on mild steel, Appl. Surf. Sci. 263, 640 (2012)

22. V. Vitry, F. Delaunois, C. Dumortier, How heat treatment can give better properties to electroless nickel-boron coatings, Metall. Ital. 101(4), 2009

23. L. Bonin, Replacement of lead stabilizer in electroless Nickel-Boron baths: New composition of green baths with properties characterization, Thesis, Université de Mons, Mons, 2018

24. V. Vitry, A.-F. Kanta, J. Dille, F. Delaunois, Structural state of electroless nickel-boron deposits ( 5 wt.\% B): Characterization by XRD and TEM, Surf. Coat. Technol. 206(16), 3444 (2012)

Citation de l'article : Alex Montagne, Véronique Vitry, Luiza Bonin, Muhammad Zeeshan Mughal, Marco Sebastiani, Edoardo Bemporad, Alain Iost, Mariana Henriette Staia, Contraintes résiduelles et comportement mécanique de revêtements nickel-bore, Matériaux \& Techniques 107, 205 (2019) 